\title{
Mycological Analysis and Potential Health Hazards of Dextrose Intravenous Infusions in Nigeria.
}

\author{
Babalola, M. O. and Akinyanju, J. A. \\ ${ }^{I}$ Department of Microbiology, Adekunle Ajasin University, P.M.B. 001, Akungba Akoko, Ondo State, Nigeria . \\ ${ }^{2}$ Department of Microbiology, University of Ilorin, P.M.B. 1515, Ilorin, Nigeria.
}

\begin{abstract}
One hundred and forty two samples (142) of two brands of commercial intravenous fluids manufactured and marketed in Nigeria were analyzed for fungal contaminants. The $\mathbf{p H}$ and osmolarity values were also determined. All samples were analyzed within their expiry dates. The infusions were categorized into the visibly defective samples and the visibly normal samples. Sixty percent $(60 \%)$ of the samples were contaminated with at least one fungal species. The fungal counts ranged from $1600 \mathrm{cfu}^{-\mathrm{ml}^{-1}}$ in the visibly defective sample to 50 $\mathrm{cfu}^{-\mathrm{ml}^{-1}}$ in the visibly normal sample. Fourteen viable fungal species belonging to ten genera were isolated. The fungal isolates in the visibly defective samples were Aspergillus repens, Aspergillus glaucus, Penicillium roqueforti, Chaetomium spp, Paecilomyces variotii, Humicola grisea, Geotrichum candidum and Geomyces cretacea, while the visibly normal samples contained Aspergillus japonicus, Candida valida, Candida krusei, Candida parapsilosis, Geomyces cretacea, Paecilomyces variotii, Humicola grisea and Fusarium oxysporum. The pH and osmolarity values of contaminated samples were lower than specified for the products. [pH:4.5-5, osmolarity 280mosmol/l for 5\% Dextrose infusion; pH 6 , osmolarity $555 \mathrm{mosmol} / \mathrm{l}$ for $10 \%$ Dextrose infusion ] The presence of pathogenic fungi particularly in the visibly normal samples before their expiry dates, coupled with the reductions in $\mathrm{pH}$ and osmolarity values below the critical safe levels $\left(\mathrm{pH} \mathrm{4,} \mathrm{\&} 306 \mathrm{mosmol}^{-1}\right.$ ) render the two brands and two categories of products potentially hazardous to health.
\end{abstract}

\section{INTRODUCTION}

The parenteral route of administration is generally adopted for medicaments that cannot be given orally, either because of patients' intolerance, drug instability, or poor absorption via the enteral route. In the unconscious patient, parenteral administration is the only safe and most effective means of administering medicaments through the intravenous route ( The Pharmaceutical Codex, 1994). Sterile intravenous infusion fluids, popularly called "Drips", are large volume parenteral products which consists of single dose injections with a volume of 100 to $1000 \mathrm{ml}$.

It is generally mandatory that microorganisms or their products must not be present in sterile pharmaceutical products throughout its shelf life (PHLS Working Party, 1971; European Pharmacopoeia, 2000). Pharmaceutical preparations are regarded contaminated or spoilt, if, low levels of acutely pathogenic microorganisms or higher levels of opportunist pathogens are present, if toxic microbial metabolites persist even after death or removal of any microorganism originally present, or if chemical changes have occurred in the product(Hugo and Russell, 1992).

Although Pharmaceutical Manufacturers strive under Good Manufacturing Practice (GMP) to ensure quality of their products, occasionally this is not achieved due to inadequate analytical facilities, lack of properly trained personnel, or indeterminate errors during quality control process (Aluoch-Orwa, et al., 1995). Contamination is a recurrent problem and can have fatal consequences particularly with intravenous products. In a Nigerian survey of intravenous products by the National Agency for Food Drug Administration and Control (NAFDAC) in 2004, out of 566 samples comprising 42 brands from 8 manufacturers, 9 brands of Dextrose $5 \%$, Dextrose $4.3 \%$ in $0.18 \% \mathrm{NaCl}$, Darrows half strength, and Dextrose $50 \%$ failed the microbiological specification for intravenous fluids, thereby prompting NAFDAC to instititute recall of the contaminated products (Atata et al., 2007).

Microbial contamination of injections and infusions often result from poor sterilization management, obsolete equipment, inappropriate production environment, and poor quality packaging (Caudron et al., 2008). Parenteral nutritions and Intravenous fat emulsions can become contaminated during preparation and infusion, with fungal pathogens (Kuwahara, et al., 2010) especially Candida species which accounts for $20-30 \%$ of systemic infections associated with Central venous catheters. Candida albicans has the ability to either grow very well or sustain prolonged viability in all nutritional intravenous products. Infusion fluids requiring 
compounding or the addition of medications to the fluid container were found to produce $7 \%$ of primary bloodstream infections when those fluids were prepared (Macias et al., 2010).

Septicemia arising from the administration of contaminated fluids is a particular complication and concern in intravenous therapy. Organisms which have been associated with infusion septicemia include Staphylococcus, Klebsiella, Aspergillus, and Candida. The genus Candida has been incriminated as one of the notorious contaminants of infusion fluids giving rise to fungal septicemia after infusion therapy(Goldman et al., 1993). Candida krusei is a commonly isolated opportunist pathogen responsible for serious septic infections in susceptible patients (McQuillen et al., 1994). In the year 2005 and 2006, administration of Candida albicans contaminated parenteral fluid was responsible for a major outbreak of systemic candidosis in India (Kumar et al., 2011). Pathogenic agents have been reported transmitted by direct contact or indirectly via contaminated instruments and intravenous fluids with fungal colonization and invasive candidosis in babies at Neonatal Intensive Care Units (Rao et al., 2005). For more than two decades, Candida albicans and C. parapsilosis have been recognized to pose significant danger to preterm infants, causing candidemia, particularly catheter related, bloodstream infections, with a crude mortality rate of $23 \%$ to $50 \%$. The risk factors include prematurity, central venous catheters, parenteral nutrition, intravenous fluids and intubation (Polin and Siman, 2003). Recently, Vagna and Henao (2010) described the manifestations of meningitis by Candida to include disseminated Candidiasis in premature infants, ventricular drainage devices infections and chronic isolated meningitis which were directly related to access of Candida albicans, Candida parapsilosis and Candida tropicalis to the Central Nervous System through the bloodstream.

In $90 \%$ of patients with fungemia and endocarditis owing to infection with the genus Paecilomyces, the predisposing factors were found to be peritoneal dialysis, contaminated intravenous fluids and medicaments (Marzec and Heron, 1993). The high glucose concentration of both dextrose infusions and dialysate fluids render them optimal media for fungi growth, even in dialysate fluids where Paecilomyces variotii was isolated and incriminated as the aetiology of peritonitis in a patient (Elamin et al., 2010 )

Acute systemic infections and deaths have resulted from the administration of contaminated fluids where invasive infections caused by fungi were the major causes of morbidity and mortality in the immunocompromised patient (Cornelius, et al., 1998). The Reuters News reported the death of nine patients in an Alabama hospital as a result of outbreaks of infection from a batch of contaminated intravenous fluids (Peggy, 2011). In addition sixteen pregnant women were reported dead in an India Hospital after the administration of contaminated dextrose intravenous fluids that led to infection, excessive bleeding and multi organ failure (Rajalakshmi, 2011)

In the tropical countries such as Nigeria, pharmaceutical preparations are frequently stored under conditions of high temperature $\left(\mathrm{Av}=31^{\circ} \mathrm{C}\right)$ and high relative humidity $(\mathrm{Av}=75 \%)$; and may be dispensed in non - protective packaging (Blair, et al., 1998). While the presence of a few microbial survivors in an injection after production is often unlikely to induce infection, considerable growth can occur during storage prior to administration thereby yielding highly infective or toxic products( Hugo and Russell, 1992). While these products were intended to mitigate diseases and death, more infections and deaths are rather often initiated worldwide, most especially in developing countries, by such unwholesome drugs. Although Aluoch- Orwa et al., 1995 reported substantial failure in the available content of the active ingredients of some commercial intravenous infusions in Kenya, Atata et al., 2007 reported on the microbiological qualities of some intravenous fluids in Nigeria. However, important parameters of $\mathrm{pH}$ and osmolarity values of the products were not determined in both studies.

The objective of this research was to evaluate the presence of fungi in commercial 5\% and $10 \%$ Dextrose intravenous fluids retailed under tropical conditions, and to evaluate the changes in $\mathrm{pH}$ and osmolarity values of the products that may have been caused by the fungal contamination.

\section{Samples}

\section{MATERIALS AND METHODS}

A total of 142 units of two brands of 5\% (100) and 10\% (42) dextrose intravenous fluids manufactured and marketed in Nigeria were randomly purchased from various retail outlets. The samples of each brand was made up of $50 \%$ visibly defective units and 50\% visibly normal units.

\section{Fungal Content Analyses of the Samples}

Each of the visibly defective samples was analyzed employing the pour plate technique following the methods of Scott et al. (1985) and adaptation of the British Pharmacopoeia (1988) in consistence with the U.S. Pharmacopoeia (2008). Each sample consist of a $500 \mathrm{ml}$ intravenous infusion in a plastic bag. The exterior of the bag was disinfected by swabbing with $70 \%$ ethanol. The content was shaken properly and with the aid of a sterile syringe inserted through the wall of the bag, $10 \mathrm{ml}$ sample of each product was withdrawn and diluted ten-fold in sterile distilled water. One $\mathrm{ml}$ of the aliquot was used to seed replicate plates of sterile Sabouraud Dextrose Agar (SDA) supplemented with $0.025 \mathrm{~g}$ Streptomycin. The plates were incubated at $27^{\circ} \mathrm{C}$ for 5 days. 
Mycological analysis and potential health hazards of dextrose intravenous.....

Under a laminar air flow cabinet, the membrane filtration technique was employed to analyze the visibly normal samples. The whole content of $500 \mathrm{ml}$ bag was filtered through a $0.45 \mu \mathrm{m}$ membrane filter, coupled on a sterile $47 \mathrm{~mm}$ magnetic filter funnel(Gelman Sciences, USA). After filtration, the membrane filter was cut aseptically into two and incubated in sterile Sabouraud Broth supplemented with $0.025 \mathrm{~g}$ Streptomycin at $27^{0} \mathrm{C}$ for 5 days. At the end of the fifth day, $1 \mathrm{ml}$ aliquot of the culture was inoculated into replicates sterile plates of SDA supplemented with $0.025 \mathrm{~g}$ Streptomycin and incubated at $27^{\circ} \mathrm{C}$ for 5 days. The resultant colonies were enumerated and the cultural characteristics recorded. Pure cultures were developed and stored on SDA slants as stock cultures which were recultured every two weeks until employed for identification.

Stringent aseptic techniques were adopted to prevent adventitious contamination of the work. Negative controls employing sterile distilled water in place of infusions were set up.

$\mathrm{PH}$ and osmolarity determination

The $\mathrm{pH}$ of the samples were measured using a Pye - Unicam pH meter model $291 \mathrm{mkz}$ equipped with a glass electrode (accuracy, $\pm 0.01 \mathrm{pH}$ unit).

The osmolarity value of each test sample was determined using an advanced Osmometer 3W11.

\section{Characterization and Identification of the fungal isolates}

Typical discrete colonies of each isolate were selected and studied employing colonial and cultural characteristics. Yeast isolates were subjected to a number of biochemical tests and cultured for pseudo mycelium formation (Lodder, 1970). The isolates were further identified based on the microscopic fungal features such as phialides, septate sporangiophores, conidiophores and budding, as previously described (Harrigan and McCance, 1976). Furthermore, typical isolates were confirmed by culturing the pure isolates on 2\% Malt Extract Agar to achieve enough conidiation, from which suspensions were prepared and inoculated into the BIOLOG FF Microplate (BIOLOG, CA, USA) and incubated at $26^{\circ} \mathrm{C}$ for 5days. Characteristic fingerprints of each isolate were then read using the BIOLOG Microstation and confirmed with the FF Database.

\section{RESULTS AND DISCUSSION}

The attributes of the samples, the visible defects, the fungal counts and species are summarized in Tables $1 \mathrm{a}, 1 \mathrm{~b}, 1 \mathrm{c}$ and $1 \mathrm{~d}$. While the products carry comparable production dates, none had expired prior to analysis. A significant higher number of fungal counts were observed in the visibly defective samples than the visibly normal samples in both categories of products. However, Aspergillus and Penicillium were not recovered from the visibly normal samples of $10 \%$ Dextrose products (Tables 2 and 3 ).

Table 1a: Attributes and fungal species isolated from Defective 5\% Dextrose intravenous fluids.

\begin{tabular}{|c|c|c|c|c|c|}
\hline$\overline{\text { Product }}$ & $\begin{array}{l}\text { Age of } \\
\text { Sample }\end{array}$ & $\begin{array}{lr}\text { Expiry } & \text { date } \\
\text { (Months } & \text { to } \\
\text { expiry) } & \\
\end{array}$ & $\begin{array}{l}\text { Visible } \\
\text { defect }\end{array}$ & $\begin{array}{l}\text { Fungal } \\
\text { Count }(\mathrm{cfu} / \mathrm{ml})\end{array}$ & Fungal Species Isolated \\
\hline DF1 & 15 months & Sept.2004(20) & $\begin{array}{l}\text { Whitish } \\
\text { strands }\end{array}$ & 270 & $\begin{array}{l}\text { Aspergillus repens, Geomyces } \\
\text { cretacea, Paecilomyces variotii }\end{array}$ \\
\hline DF2 & 12 months & Jan. 2005(24) & $\begin{array}{l}\text { Brown } \\
\text { coloration }\end{array}$ & 630 & $\begin{array}{l}\text { Aspergillus glaucus, Penicillium } \\
\text { roqueforti, Aspergillus repens, } \\
\text { Paecilomyces variotii }\end{array}$ \\
\hline DF3 & 17 months & Jul.2004(19) & Cloudy & 320 & $\begin{array}{l}\text { Geomyces cretacea, Paecilomyces } \\
\text { variotii, Penicillium roqueforti }\end{array}$ \\
\hline DF4 & 17 months & Jul. 2004(19) & $\begin{array}{l}\text { Wooly } \\
\text { strands }\end{array}$ & 300 & $\begin{array}{l}\text { Paecilomyces variotii Aspergillus } \\
\text { glaucus, Penicillium roqueforti }\end{array}$ \\
\hline DF5 & 16 months & Aug. 2004(20) & $\begin{array}{l}\text { Wooly } \\
\text { strands }\end{array}$ & 300 & $\begin{array}{l}\text { Paecilomyces variotii Aspergillus } \\
\text { glaucus, Geomyces cretacea }\end{array}$ \\
\hline DF6 & 18 months & Jun. 2004(18) & $\begin{array}{l}\text { Tainted } \\
\text { wooly } \\
\text { strands }\end{array}$ & 160 & $\begin{array}{l}\text { Paecilomyces variotii Aspergillus } \\
\text { repens, Penicillium roqueforti }\end{array}$ \\
\hline DF7 & 18 months & Jun. 2004(18) & $\begin{array}{l}\text { Brownish } \\
\text { strands }\end{array}$ & 330 & $\begin{array}{l}\text { Aspergillus glaucus, Aspergillus } \\
\text { repens, Geomyces cretacea, } \\
\text { Paecilomyces variotii }\end{array}$ \\
\hline DF8 & 18 months & Jun. 2004(18) & Cloudy & 350 & $\begin{array}{l}\text { Geomyces cretacea, Paecilomyces } \\
\text { variotii, Penicillium roqueforti }\end{array}$ \\
\hline DF9 & 18 months & Jun 2004(18) & $\begin{array}{l}\text { Wooly } \\
\text { strands }\end{array}$ & 300 & $\begin{array}{l}\text { Aspergillus glaucus, Aspergillus } \\
\text { repens, Paecilomyces variotii, } \\
\text { Penicillium roqueforti }\end{array}$ \\
\hline DF10 & 18 months & Jun. 2004(18) & Ropiness & 250 & Geomyces cretacea Penicillium \\
\hline
\end{tabular}


Mycological analysis and potential health hazards of dextrose intravenous.....

\begin{tabular}{|c|c|c|c|c|c|}
\hline & & & & & roqueforti \\
\hline DF11 & 18 months & Jun 2004(18) & Cloudy & 248 & $\begin{array}{l}\text { Aspergillus repens, Geomyces } \\
\text { cretacea, Paecilomyces variotii, } \\
\text { Penicillium roqueforti }\end{array}$ \\
\hline DF12 & 19 months & Jul. 2004(17) & $\begin{array}{l}\text { Brown } \\
\text { coloration }\end{array}$ & 358 & $\begin{array}{l}\text { Aspergillus glaucus. } \\
\text { repens, Gergillus } \\
\text { Paecilomyces variotii }\end{array}$ \\
\hline DF13 & 19 months & Jul. 2004(17) & $\begin{array}{l}\text { Brown } \\
\text { coloration }\end{array}$ & 320 & $\begin{array}{l}\text { Aspergillus glaucus, Aspergillus } \\
\text { repens, Geomyces cretacea }\end{array}$ \\
\hline DF14 & 19 months & Jul. 2004(17) & $\begin{array}{l}\text { Whitish } \\
\text { strands }\end{array}$ & 300 & $\begin{array}{l}\text { Aspergillus glaucus, Geomyces } \\
\text { cretacea, Paecilomyces variotii }\end{array}$ \\
\hline DF15 & 19 months & Jul. 2004(17) & $\begin{array}{l}\text { Brownish } \\
\text { slimy } \\
\text { strands }\end{array}$ & 350 & $\begin{array}{l}\text { Aspergillus repens, Geomyces } \\
\text { cretacea, Penicillium roqueforti }\end{array}$ \\
\hline DF16 & 19 months & Jul. 2004(17) & $\begin{array}{l}\text { Whitish } \\
\text { strands }\end{array}$ & 248 & $\begin{array}{l}\text { Aspergillus glaucus, Geomyces } \\
\text { cretacea, Paecilomyces variotii }\end{array}$ \\
\hline DF17 & 12 months & Feb. 2005(24) & $\begin{array}{l}\text { Brownish } \\
\text { strands }\end{array}$ & 320 & $\begin{array}{l}\text { Aspergillus glaucus, Aspergillus } \\
\text { repens }\end{array}$ \\
\hline DF18 & 20 months & Aug. 2004(16) & $\begin{array}{l}\text { Brownish } \\
\text { strands }\end{array}$ & 300 & $\begin{array}{l}\text { Asperillus glaucus, } \\
\text { repens, } \quad \text { Geomyces } \\
\text { Paecilomyces variotilii }\end{array}$ \\
\hline DF19 & 12 months & Feb. 2005(24) & $\begin{array}{l}\text { Packaging } \\
\text { defect }\end{array}$ & 0 & Nil \\
\hline DF20 & 20 months & Aug, 2004(16) & $\begin{array}{l}\text { Brown } \\
\text { coloration }\end{array}$ & 350 & $\begin{array}{l}\text { Aspergillus glaucus, } \\
\text { repens, Gergillus } \\
\text { Paecilomyces variotii }\end{array}$ \\
\hline DF21 & 20 months & Aug. 2004(16) & Bloated & 0 & Nil \\
\hline DF22 & 20 months & Aug.2004(16) & Packaging & 0 & Nil \\
\hline DF23 & 20 months & Aug, 2004(16) & Cloudy & 330 & $\begin{array}{l}\text { Aspergillus glaucus, Geomyces } \\
\text { cretacea, Paecilomyces variotii, } \\
\text { Penicillium roqueforti }\end{array}$ \\
\hline DF24 & 20 months & Aug. 2004(16) & Less content & 230 & $\begin{array}{l}\text { Aspergillus glaucus, Geomyces } \\
\text { cretacea, Paecilomyces variotii }\end{array}$ \\
\hline DF25 & 12 months & Feb. 2005(24) & Less content & 0 & Nil \\
\hline DF26 & 24 months & Dec 2008(12) & Cloudy & 340 & $\begin{array}{l}\text { Aspergillus glaucus, Geomyces } \\
\text { cretacea, Paecilomyces variotii, } \\
\text { Penicillium roqueforti }\end{array}$ \\
\hline DF27 & 24 months & Dec.2008(12) & $\begin{array}{l}\text { Brownish } \\
\text { coloration }\end{array}$ & 360 & $\begin{array}{l}\text { Aspergillus glaucus, Aspergillus } \\
\text { repens, Geomyces cretacea, } \\
\text { Penicillium roqueforti }\end{array}$ \\
\hline DF28 & 24 months & Aug. 2009(12) & $\begin{array}{l}\text { Brownish } \\
\text { coloration }\end{array}$ & 220 & $\begin{array}{l}\text { Aspergillus glaucus, Aspergillus } \\
\text { repens, Paecilomyces variotii }\end{array}$ \\
\hline DF29 & 24 months & Aug. 2009(12) & $\begin{array}{l}\text { Tainted } \\
\text { strands }\end{array}$ & 180 & $\begin{array}{l}\text { Aspergillus repens, Geomyces } \\
\text { cretacea, Paecilomyces variotii }\end{array}$ \\
\hline DF30 & 24 months & Aug.2009(12) & Bloated & 0 & Nil \\
\hline$\overline{\text { DF31 }}$ & 25 months & Sep 2009(11) & $\begin{array}{l}\text { Brownish } \\
\text { strands }\end{array}$ & 200 & $\begin{array}{l}\text { Aspergillus glaucus, Aspergillus } \\
\text { repens, Penicilliumroqueforti }\end{array}$ \\
\hline DF32 & 24 months & Aug. 2009(12) & $\begin{array}{l}\text { Whitish } \\
\text { strands }\end{array}$ & 240 & $\begin{array}{l}\text { Aspergillus glaucus, Geomyces } \\
\text { cretacea, Paecilomyces variotii, } \\
\text { Penicillium roqueforti }\end{array}$ \\
\hline DF33 & 16 months & Oct 2010(20) & $\begin{array}{l}\text { Brownish } \\
\text { strands }\end{array}$ & 230 & $\begin{array}{l}\text { Aspergillus glaucus, Aspergillus } \\
\text { repens, Paecilomyces variotii, } \\
\text { Penicillium roqueforti }\end{array}$ \\
\hline DF34 & 14 months & Sep 2010(22) & $\begin{array}{l}\text { Tainted } \\
\text { speckles }\end{array}$ & 120 & $\begin{array}{l}\text { Aspergillus glaucus, Aspergillus } \\
\text { repens, }\end{array}$ \\
\hline
\end{tabular}


Mycological analysis and potential health hazards of dextrose intravenous.....

\begin{tabular}{|c|c|c|c|c|c|}
\hline DF35 & 17 months & Nov 2010(19) & Cloudy & 220 & $\begin{array}{l}\text { Aspergillus repens, Geomyces } \\
\text { cretacea, Penicillium roqueforti }\end{array}$ \\
\hline DF36 & 15 months & Aug. 2010(21) & Less content & 0 & Nil \\
\hline DF37 & 15 months & Aug.2010(21) & Cloudy & 100 & $\begin{array}{l}\text { Paecilomyces variotii, Geomyces } \\
\text { cretacea, Penicillium roqueforti }\end{array}$ \\
\hline DF38 & 16 months & Oct 2010(20) & $\begin{array}{l}\text { Whitish } \\
\text { strands }\end{array}$ & 180 & $\begin{array}{l}\text { Aspergillus glaucus, Aspergillus } \\
\text { repens, Geomyces cretacea }\end{array}$ \\
\hline DF39 & 16 months & Oct 2010(20) & $\begin{array}{l}\text { Tainted } \\
\text { strands }\end{array}$ & 220 & $\begin{array}{l}\text { Aspergillus glaucus, Aspergillus } \\
\text { repens, Geomyces cretacea, } \\
\text { Penicillium roqueforti }\end{array}$ \\
\hline DF40 & 12 months & Oct 2012(24) & Less content & 0 & Nil \\
\hline DF41 & 33 months & Nov 2010(3) & Dark strands & 420 & $\begin{array}{l}\text { Aspergillus glaucus, Penicillium } \\
\text { roqueforti, Paecilomyces variotii }\end{array}$ \\
\hline DF42 & 28 months & Apr 2011(8) & $\begin{array}{l}\text { Whitish } \\
\text { strands }\end{array}$ & 300 & $\begin{array}{l}\text { Aspergillus glaucus, Aspergillus } \\
\text { repens, Geomyces cretacea, } \\
\text { Paecilomyces variotii }\end{array}$ \\
\hline$\overline{D F} 43$ & 31 months & Feb 2011(6) & $\begin{array}{l}\text { Faint milky } \\
\text { coloration }\end{array}$ & 330 & $\begin{array}{l}\text { Aspergillus repens, Geomyces } \\
\text { cretacea, Paecilomyces variotii, } \\
\text { Penicillium roqueforti }\end{array}$ \\
\hline DF44 & 29 months & Mar 2011(7) & $\begin{array}{l}\text { Whitish } \\
\text { strands }\end{array}$ & 320 & $\begin{array}{l}\text { Aspergillus glaucus, Aspergillus } \\
\text { repens, Paecilomyces variotii, } \\
\text { Penicillium roqueforti }\end{array}$ \\
\hline DF45 & 29 months & Mar 2011(7) & $\begin{array}{l}\text { Whitish } \\
\text { strands }\end{array}$ & 320 & $\begin{array}{l}\text { Aspergillus glaucus, Aspergillus } \\
\text { repens, Geomyces cretacea }\end{array}$ \\
\hline DF46 & 31 months & Feb 2011(5) & $\begin{array}{l}\text { Whitish } \\
\text { strands }\end{array}$ & 330 & $\begin{array}{l}\text { Aspergillus repens, Paecilomyces } \\
\text { variotii, Penicillium roqueforti }\end{array}$ \\
\hline DF47 & 31 months & Feb 2011(5) & $\begin{array}{l}\text { Whitish } \\
\text { strands }\end{array}$ & 300 & $\begin{array}{l}\text { Aspergillus glaucus, Aspergillus } \\
\text { repens, Geomyces cretacea }\end{array}$ \\
\hline DF48 & 29 months & Mar 2011(7) & $\begin{array}{l}\text { Yellowish } \\
\text { coloration }\end{array}$ & 320 & $\begin{array}{l}\text { Aspergillus glaucus, Aspergillus } \\
\text { repens, Geomyces cretacea, } \\
\text { Paecilomyces variotii }\end{array}$ \\
\hline
\end{tabular}


Mycological analysis and potential health hazards of dextrose intravenous.....

\begin{tabular}{|l|l|l|l|l|l|}
\hline DF49 & 28 months & Apr 2011(8) & Less content & 128 & $\begin{array}{l}\text { Aspergillus repens, Paecilomyces } \\
\text { variotii, }\end{array}$ \\
\hline DF50 & 29 months & Mar 2011(7) & Less content & 240 & $\begin{array}{l}\text { Aspergillus repens, Geomyces } \\
\text { cretacea, Penicillium roqueforti }\end{array}$ \\
\hline
\end{tabular}

Table 1b: Attributes and fungal species isolated from Normal 5\% Dextrose intravenous fluids.

\begin{tabular}{|c|c|c|c|c|c|}
\hline Product & $\begin{array}{l}\text { Age of } \\
\text { Sample }\end{array}$ & $\begin{array}{l}\text { Expiry date } \\
\text { (months to expiry) }\end{array}$ & $\begin{array}{l}\text { Visible } \\
\text { defect }\end{array}$ & $\begin{array}{l}\text { Fungal } \\
\text { Count }(\mathrm{cfu} / \mathrm{ml})\end{array}$ & Fungal Species Isolated \\
\hline NF1 & 15 months & Sept.2004((21) & Nil & 150 & $\begin{array}{lr}\text { A. japonicus, } & \text { Fusarium } \\
\text { oxysporum, } & \text { Paecilomyces } \\
\text { variotii } & \end{array}$ \\
\hline NF2 & 3 months & Sept.2005(33) & Nil & 10 & Candida krusei \\
\hline NF3 & 17 months & Jul.2004(19) & Nil & 60 & $\begin{array}{lr}\text { Paecilomyces variotii, } & A . \\
\text { japonicus, } & \text { Candida } \\
\text { krusei } & \end{array}$ \\
\hline NF4 & 17 months & Jul.2004(19) & Nil & 50 & $\begin{array}{l}\text { Paecilomyces variotii, } \\
\text { japonicus }\end{array}$ \\
\hline NF5 & 15 months & Aug. 2004(21) & Nil & 50 & $\begin{array}{l}\text { Paecilomyces } \\
\text { Geomyces cretacea }\end{array}$ \\
\hline NF6 & 18 months & Jun. 2004(18) & Nil & 150 & $\begin{array}{lr}\text { Paecilomyces } & \text { variotii, } \\
\text { A.japonicus, } & \text { Candida krusei }\end{array}$ \\
\hline NF7 & 18 months & Jun. 2004(18) & Nil & 60 & $\begin{array}{l}\text { A. japonicus ,Candida krusei, } \\
\text { Paecilomyces variotii }\end{array}$ \\
\hline NF8 & 18 months & Jun. 2004(18) & Nil & 30 & $\begin{array}{l}\text { Candida krusei, Paecilomyces } \\
\text { variotii }\end{array}$ \\
\hline NF9 & 17 months & Jul.2004(19) & Nil & 30 & $\begin{array}{lll}\text { Candida } & \text { krusei, } & \text { Geomyces } \\
\text { cretacea }\end{array}$ \\
\hline NF10 & 17 months & Jul.2004(19) & Nil & 40 & A. japonicus, Candida krusei \\
\hline NF11 & 18 months & Jun.2004(18) & Nil & 50 & A. japonicus, Candida krusei \\
\hline NF12 & 19 months & Jul. 2004(17) & Nil & 40 & $\begin{array}{ll}\text { Candida } & \text { krusei, } \\
\text { cretacea }\end{array}$ \\
\hline NF13 & 19 months & Jul.2004(17) & Nil & 50 & A. japonicus, Candida krusei \\
\hline NF14 & 19 months & Jul. 2004(17) & Nil & 60 & A.japonicus, Candida krusei \\
\hline NF15 & 19 months & Jul. 2004(17) & Nil & 70 & $\begin{array}{l}\text { A. japonicus, Candida krusei, } \\
\text { Fusarium oxysporum }\end{array}$ \\
\hline NF16 & 19 months & Jul.2004(17) & Nil & 70 & A. japonicus, Candida krusei \\
\hline NF17 & 12 months & Feb. 2005(24) & Nil & 0 & Nil \\
\hline NF18 & 20 months & Aug. 2005(16) & Nil & 70 & A. japonicus, Candida krusei \\
\hline NF19 & 12 months & Feb.2005(24) & Nil & 20 & $\begin{array}{ll}\text { Geomyces } & \text { cretacea, } \\
\text { Paecilomyces variotii } & \end{array}$ \\
\hline NF20 & 20 months & Aug. 2004(16) & Nil & 60 & A. japonicus, Candida krusei \\
\hline NF21 & 20months & Aug. 2004(16) & Nil & 70 & A. japonicus, Candida krusei \\
\hline NF22 & 20 months & Aug 2004(16) & Nil & 60 & A. japonicus, Candida krusei \\
\hline NF23 & 20 months & Aug 2004(16) & Nil & 50 & $\begin{array}{ll}\text { Candida } & \text { krusei, } \\
\text { cretacea }\end{array}$ \\
\hline NF24 & 20 months & Aug 2004(16) & Nil & 40 & $\begin{array}{ll}\text { Geomyces } & \text { cretacea, } \\
\text { Paecilomyces variotii } & \\
\end{array}$ \\
\hline NF25 & 12 months & Feb. 2005(24) & Nil & 0 & Nil \\
\hline NF26 & 24 months & Dec.2008(12) & Nil & 80 & $\begin{array}{l}\text { A. japonicus, Candida krusei, } \\
\text { Fusarium oxysporum }\end{array}$ \\
\hline NF27 & 24 months & Dec.2008(12) & Nil & 80 & $\begin{array}{l}\text { A. japonicus, Candida krusei, } \\
\text { Geomyces cretacea }\end{array}$ \\
\hline NF28 & 24 months & Aug 2009(12) & Nil & 70 & $\begin{array}{l}\text { A. japonicus, Candida krusei, } \\
\text { Fusarium oxysporum }\end{array}$ \\
\hline
\end{tabular}


Mycological analysis and potential health hazards of dextrose intravenous.....

\begin{tabular}{|c|c|c|c|c|c|}
\hline NF29 & 24 months & Aug 2009(12) & Nil & 90 & $\begin{array}{l}\text { A. japonicus, Candida krusei, } \\
\text { Fusarium oxysporum }\end{array}$ \\
\hline NF30 & 24 months & Aug 2009(12) & Nil & 80 & $\begin{array}{l}\text { A. japonicus, Candida krusei, } \\
\text { Fusarium oxysporum }\end{array}$ \\
\hline NF31 & 25 months & Sep.2009(11) & Nil & 90 & $\begin{array}{l}\text { A. japonicus, Candida krusei, } \\
\text { Fusarium oxysporum, }\end{array}$ \\
\hline NF32 & 24 months & Aug 2009(12) & Nil & 80 & $\begin{array}{l}\text { A. japonicus, Candida krusei, } \\
\text { Fusarium oxysporum }\end{array}$ \\
\hline NF33 & 16 months & Oct.2010(20) & Nil & 60 & A. japonicus, Candida krusei \\
\hline NF34 & 14 months & Dec.2010(22) & Nil & 50 & $\begin{array}{l}\text { Fusarium oxysporum, Geomyces } \\
\text { cretacea }\end{array}$ \\
\hline NF35 & 17 months & Nov.2010(19) & Nil & 60 & A. japonicus, Candida krusei \\
\hline NF36 & 15 months & Aug. 2010(21) & Nil & 60 & $\begin{array}{ll}\text { Geomyces } & \text { cretacea, } \\
\text { Paecilomyces variotii } & \end{array}$ \\
\hline NF37 & 15 months & Aug.2010(21) & Nil & 50 & $\begin{array}{l}\text { Fusarium oxysporum, } \\
\text { Paecilomyces variotii }\end{array}$ \\
\hline NF38 & 14 months & Dec 2010(22) & Nil & 50 & $\begin{array}{l}\text { Paecilomyces variotii, Fusarium } \\
\text { oxysporum }\end{array}$ \\
\hline NF39 & 16 months & Oct. 2010(20) & Nil & 60 & $\begin{array}{l}\text { Fusarium oxysporum, Geomyces } \\
\text { cretacea, Paecilomyces variotii }\end{array}$ \\
\hline NF40 & 13 months & Nov.2012(23) & Nil & 50 & $\begin{array}{ll}\text { Geomyces } & \text { cretacea, } \\
\text { Paecilomyces variotii } & \end{array}$ \\
\hline NF41 & 28 months & Apr.2011(8) & Nil & 80 & $\begin{array}{l}\text { A. japonicus, Paecilomyces } \\
\text { variotii Fusarium oxysporum }\end{array}$ \\
\hline NF42 & 27 months & May 2011(9) & Nil & 70 & $\begin{array}{l}\text { A. japonicus, Candida krusei, } \\
\text { Paecilomyces variotii }\end{array}$ \\
\hline NF43 & 26 months & Jun.2011(10) & Nil & 70 & $\begin{array}{l}\text { A. japonicus, Paecilomyces } \\
\text { variotii, Candida krusei }\end{array}$ \\
\hline NF44 & 25 months & Jul.2011(11) & Nil & 60 & $\begin{array}{lr}\text { A. japonicus, } & \text { Fusarium } \\
\text { oxysporum, } & \text { Paecilomyces } \\
\text { variotii } & \end{array}$ \\
\hline NF45 & 24 months & Aug.2011(12) & Nil & 60 & $\begin{array}{l}\text { Fusarium oxysporum, } \\
\text { Paecilomyces variotii }\end{array}$ \\
\hline NF46 & 30 months & Mar 2011(6) & Nil & 70 & $\begin{array}{l}\text { A. japonicusGeomyces cretacea, } \\
\text { Paecilomyces variotii }\end{array}$ \\
\hline NF47 & 3 months & Apr 2013(33) & Nil & 0 & Nil \\
\hline NF48 & 6 months & Jan 2013(30) & Nil & 0 & Nil \\
\hline NF49 & 7 months & Dec. 2012(29) & Nil & 20 & $\begin{array}{l}\text { Geomyces cretacea, Candida } \\
\text { krusei }\end{array}$ \\
\hline NF50 & 5 months & Sep.2013(31) & Nil & 0 & Nil \\
\hline
\end{tabular}

Table 1c: Attributes and fungal species isolated from Defective 10\% Dextrose intravenous fluids.

\begin{tabular}{|l|l|l|l|l|l|}
\hline Product & $\begin{array}{l}\text { Age of } \\
\text { Sample }\end{array}$ & $\begin{array}{l}\text { Expiry } \\
\text { date(months to } \\
\text { expiry) }\end{array}$ & $\begin{array}{l}\text { Visible } \\
\text { defect }\end{array}$ & $\begin{array}{l}\text { Fungal } \\
\text { Count(cfu/ml) }\end{array}$ & Fungal Species Isolated \\
\hline DT1 & 32 months & Apr.2003(4) & $\begin{array}{l}\text { Dark } \\
\text { Wooly } \\
\text { strands }\end{array}$ & 1600 & $\begin{array}{l}\text { Exophiala dermantitidis, } \\
\text { Geomyces } \\
\text { Chaetacea, }, \\
\text { candidum }\end{array}$ \\
\hline DT2 & 32 months & Aug. 2003(4) & $\begin{array}{l}\text { Wooly } \\
\text { strands }\end{array}$ & 250 & $\begin{array}{l}\text { Candida krusei, Chaetomium } \\
\text { sp, Exophiala dermantitidis, } \\
\text { Geotrichum candidum }\end{array}$ \\
\hline DT3 & 30 months & Apr. 2003(6) & Cloudy & 110 & $\begin{array}{l}\text { Geomyces cretacea, } \\
\text { Chaetomium sp, Geotrichum } \\
\text { candidum }\end{array}$ \\
\hline DT4 & 32 months & Jul.2004(4) & $\begin{array}{l}\text { Whitish } \\
\text { strands }\end{array}$ & 120 & $\begin{array}{l}\text { Humicola grisea, Chaetomium } \\
\text { sp, Geomyces } \\
\text { Geotrichum candidum }\end{array}$ \\
\hline
\end{tabular}


Mycological analysis and potential health hazards of dextrose intravenous.....

\begin{tabular}{|c|c|c|c|c|c|}
\hline DT5 & 29 months & Jul..2004(7) & Cloudy & 100 & $\begin{array}{l}\text { Geotrichum } \\
\text { Geomyces cretacea }\end{array}$ \\
\hline DT6 & 28 months & Aug.2004(8) & $\begin{array}{l}\text { Wooly } \\
\text { strands }\end{array}$ & 210 & $\begin{array}{lr}\text { Candida krusei, } & \text { Exophiala } \\
\text { dermantitidis, } & \text { Geomyces } \\
\text { cretacea, } & \text { Geotrichum } \\
\text { candidum } & \end{array}$ \\
\hline DT7 & 28 months & Sept 2004(8) & Ropiness & 90 & $\begin{array}{l}\text { Candida krusei, Exophiala } \\
\text { dermantitidis }\end{array}$ \\
\hline DT8 & 28 months & Oct.2004(8) & $\begin{array}{l}\text { Slimy } \\
\text { strands }\end{array}$ & 100 & $\begin{array}{l}\text { Candida krusei, Exophiala } \\
\text { dermantitidis }\end{array}$ \\
\hline DT9 & 28 months & Nov 2008(8) & $\begin{array}{l}\text { Wooly } \\
\text { strands }\end{array}$ & 110 & $\begin{array}{lr}\text { Geomyces } & \text { cretacea, } \\
\text { Chaetomium } & \text { sp, } \\
\text { dermantitidis, } & \text { Geotrichiala } \\
\text { candidum } & \\
\end{array}$ \\
\hline DT10 & 24 months & Aug.2008(12) & Cloudy & 108 & $\begin{array}{l}\text { Candida krusei, } \text { Geomyces } \\
\text { cretacea }\end{array}$ \\
\hline DT11 & 24 months & Aug.2008(12) & Cloudy & 100 & 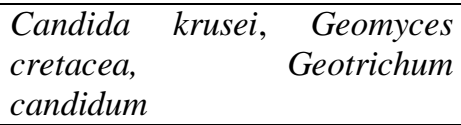 \\
\hline DT12 & 18 months & Aug.2008(18) & Cloudy & 80 & $\begin{array}{l}\text { Candida krusei, Exophiala } \\
\text { dermantitidis }\end{array}$ \\
\hline DT13 & 18 months & Aug.2009(18) & Cloudy & 120 & $\begin{array}{lrr}\begin{array}{l}\text { Candida } \\
\text { cretacea, }\end{array} & \text { krusei, } & \text { Geomyces } \\
\text { candidum } & \text { Geotrichum }\end{array}$ \\
\hline DT14 & 16 months & Sep.2009(20) & Ropiness & 80 & $\begin{array}{l}\text { Candida krusei, Exophiala } \\
\text { dermantitidis }\end{array}$ \\
\hline DT15 & 14 months & Sep.2009(22) & $\begin{array}{l}\text { Whitish } \\
\text { strands }\end{array}$ & 120 & $\begin{array}{l}\text { Humicola grisea, Chaetomium } \\
\text { sp, Geomyces cretacea, } \\
\text { Geotrichum candidum }\end{array}$ \\
\hline DT16 & 14 months & Sep.2009(22) & Cloudy & 100 & $\begin{array}{l}\text { Candida krusei, Geomyces } \\
\text { cretacea }\end{array}$ \\
\hline DT17 & 14 months & Sep.2011(22) & $\begin{array}{l}\text { Slimy } \\
\text { strands }\end{array}$ & 110 & $\begin{array}{l}\text { Candida krusei, Exophiala } \\
\text { dermantitidis }\end{array}$ \\
\hline DT18 & 14 months & Sep.2011(22) & $\begin{array}{l}\text { Whitish } \\
\text { strands }\end{array}$ & 110 & $\begin{array}{lr}\begin{array}{l}\text { Chaetomium } \\
\text { cretacea, } \\
\text { candidum }\end{array} & \begin{array}{r}\text { Geomyces } \\
\text { Geotrichum }\end{array} \\
\end{array}$ \\
\hline DT19 & 12months & Sep.2011(24) & $\begin{array}{l}\text { Wooly } \\
\text { strands }\end{array}$ & 130 & $\begin{array}{lr}\text { Geomyces cretacea, Candida } \\
\text { krusei, } \\
\text { dermantitidis, } \\
\text { candidum }\end{array}$ \\
\hline DT20 & 10 months & Oct.2011(26) & Cloudy & 80 & $\begin{array}{l}\text { Candida krusei, Chaetomium } \\
\text { sp, Exophiala dermantitidis }\end{array}$ \\
\hline DT21 & 12months & Oct. 2011(24) & $\begin{array}{l}\text { Dark } \\
\text { strands }\end{array}$ & 128 & $\begin{array}{l}\text { Geomyces cretacea Exophiala } \\
\text { dermantitidis, } \quad \text { Geotrichum } \\
\text { candidum }\end{array}$ \\
\hline
\end{tabular}

Table 1d: Attributes and fungal species isolated from Normal $10 \%$ Dextrose intravenous fluids.

\begin{tabular}{|l|l|l|l|l|l|}
\hline product & $\begin{array}{l}\text { Age of } \\
\text { Sample }\end{array}$ & $\begin{array}{l}\text { Expiry date } \\
\text { (months } \\
\text { expiry) }\end{array}$ & $\begin{array}{l}\text { Visible } \\
\text { defect }\end{array}$ & $\begin{array}{l}\text { Fungal } \\
\text { Count(cfu/ml ) }\end{array}$ & Fungal Species Isolated \\
\hline NT1 & $\begin{array}{l}32 \\
\text { months }\end{array}$ & Apr. 2003(4) & Nil & 80 & $\begin{array}{l}\text { Candida valida, Candida krusei, } \\
\text { Fusarium oxysporum, Humicola } \\
\text { grisea }\end{array}$ \\
\hline NT2 & $\begin{array}{l}30 \\
\text { months }\end{array}$ & Aug. 2003(6) & Nil & 100 & $\begin{array}{l}\text { Candida krusei, } \\
\text { oxysporum, C. } \\
\text { Humicola grisea }\end{array}$ \\
\hline NT3 & $\begin{array}{l}32 \\
\text { months }\end{array}$ & Apr.2003(4) & Nil & 110 & $\begin{array}{l}\text { Candida valida, Candida krusei, } \\
\text { C. parapsilosis, Humicola grisea }\end{array}$ \\
\hline
\end{tabular}


Mycological analysis and potential health hazards of dextrose intravenous.....

\begin{tabular}{|c|c|c|c|c|c|}
\hline NT4 & $\begin{array}{l}32 \\
\text { months }\end{array}$ & Jul.2004(4) & Nil & 60 & $\begin{array}{l}\text { Humicola grisea, C. valida } \\
\text { Fusarium oxysporum }\end{array}$ \\
\hline NT5 & $\begin{array}{l}29 \\
\text { months }\end{array}$ & Jul.2004(7) & Nil & 50 & $\begin{array}{l}\text { Candida valida, Candida krusei, } \\
\text { C. parapsilosis }\end{array}$ \\
\hline NT6 & $\begin{array}{l}28 \\
\text { months }\end{array}$ & Aug.2004(8) & Nil & 50 & $\begin{array}{l}\text { Candida valida, Candida krusei, } \\
\text { C. parapsilosis }\end{array}$ \\
\hline NT7 & $\begin{array}{l}27 \\
\text { months }\end{array}$ & Sep.2004(9) & Nil & 80 & $\begin{array}{l}\text { Fusarium oxysporum, Candida } \\
\text { valida, } C \text {. krusei }\end{array}$ \\
\hline NT8 & $\begin{array}{l}26 \\
\text { months }\end{array}$ & Oct.2004(10) & Nil & 80 & $\begin{array}{l}\text { Candida valida, Candida krusei, } \\
\text { C.parapsilosis }\end{array}$ \\
\hline NT9 & 8 months & Nov 2009(28) & Nil & 0 & Nil \\
\hline NT10 & $\begin{array}{l}24 \\
\text { months }\end{array}$ & Aug.2008(12) & Nil & 90 & $\begin{array}{l}\text { Fusarium oxysporum, Candida } \\
\text { krusei,C.parapsilosis }\end{array}$ \\
\hline NT11 & 6months & Sep..2010(30) & Nil & 0 & Nil \\
\hline NT12 & $\begin{array}{l}24 \\
\text { months }\end{array}$ & Aug.2008(12) & Nil & 80 & $\begin{array}{l}\text { Fusarium oxysporum, Humicola } \\
\text { grisea, } C \text {. valida }\end{array}$ \\
\hline NT13 & 6 months & Aug.2010(30) & Nil & 0 & Nil \\
\hline NT14 & $\begin{array}{l}12 \\
\text { months }\end{array}$ & Sep.2009(24) & Nil & 60 & $\begin{array}{l}\text { Candida krusei, Candida valida, } \\
\text { Fusarium oxysporum }\end{array}$ \\
\hline NT15 & 8 months & Sep.2009(28) & Nil & 50 & $\begin{array}{l}\text { Fusarium oxysporum, Candida } \\
\text { krusei, } \quad \text { C. parapsilosis }\end{array}$ \\
\hline NT16 & 8 months & Sep.2009(28) & Nil & 50 & $\begin{array}{l}\text { Candida } \quad \text { krusei, } \quad \text { Candida } \\
\text { valida,Candida parapsilosis }\end{array}$ \\
\hline NT17 & $\begin{array}{l}23 \\
\text { months }\end{array}$ & Oct.2011(13) & Nil & 90 & 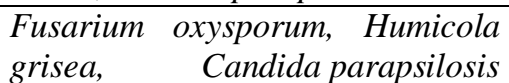 \\
\hline NT18 & $\begin{array}{l}24 \\
\text { months }\end{array}$ & Sep.2011(12) & Nil & 100 & $\begin{array}{l}\text { Fusarium oxysporum, Candida } \\
\text { valida,C.parapsilosis }\end{array}$ \\
\hline NT19 & $\begin{array}{l}24 \\
\text { months }\end{array}$ & Sep.2011(12) & Nil & 80 & $\begin{array}{l}\text { Fusarium oxysporum, } \\
\text { krusei, C.parapsilosis }\end{array}$ \\
\hline NT20 & $\begin{array}{l}24 \\
\text { months }\end{array}$ & Oct.2011(12) & Nil & 80 & $\begin{array}{l}\text { Fusarium oxysporum, Candida } \\
\text { valida, C. krusei }\end{array}$ \\
\hline NT21 & $\begin{array}{l}25 \\
\text { months }\end{array}$ & Oct.2011(11) & Nil & 90 & $\begin{array}{l}\text { Candida krusei,C.parapsilosis } \\
\text { Fusarium oxysporum }\end{array}$ \\
\hline
\end{tabular}

Table 2: Percentage occurrence of fungal isolates from $5 \%$ Dextrose intravenous fluids.

\begin{tabular}{|c|c|c|c|}
\hline \multicolumn{2}{|c|}{ Visibly defective samples } & \multicolumn{2}{|c|}{ Visibly Normal samples } \\
\hline Isolate & $\%$ occurrence & Isolate & $\%$ occurrence \\
\hline Aspergillus glaucus & $55 \%$ & Aspergillus japonicus & $60 \%$ \\
\hline Aspergillus repens & $60 \%$ & Candida krusei & $60 \%$ \\
\hline Geomyces cretacea & $60 \%$ & Fusarium oxysporum & $30 \%$ \\
\hline Paecilomyces variotii & $60 \%$ & Geomyces cretacea & $25 \%$ \\
\hline Penicillium roqueforti & $45 \%$ & Paecilomyces variotii & $40 \%$ \\
\hline
\end{tabular}

Table 3: Percentage occurrence of fungal isolates from $10 \%$ Dextrose intravenous fluids

\section{Visibly defective samples}

\begin{tabular}{lc}
\hline Isolate & \% occurrence \\
Candida krusei & $60 \%$ \\
Chaetomium spp & $40 \%$
\end{tabular}

\section{Visibly Normal samples}

$\begin{array}{lc}\text { Isolate } & \text { \% occurrence } \\ \text { Candida krusei } & 65 \% \\ \text { Candida parapsilosis } & 60 \%\end{array}$


Mycological analysis and potential health hazards of dextrose intravenous.....

Exophiala dermantitidis

Geomyces cretacea

Geotrichum candidum

Humicola grisea
$55 \%$

$65 \%$

$60 \%$

$55 \%$
Candida valida

Fusarium oxysporum

Humicola grisea
$55 \%$

$60 \%$

$30 \%$

Table 4: The pH and osmolarity values of the $5 \%$ Dextrose intravenous fluids.

\begin{tabular}{|c|c|c|c|c|c|}
\hline $\begin{array}{l}\text { Visibly } \\
\text { Defective }\end{array}$ & p H & $\begin{array}{l}\text { Osmolarity } \\
\left(\text { mosmol l }^{-1}\right)\end{array}$ & $\begin{array}{l}\text { Visibly } \\
\text { Normal } \\
\end{array}$ & $\mathbf{p} \mathbf{H}$ & $\begin{array}{l}\text { Osmolarity } \\
(\text { mosmol 1 }\end{array}$ \\
\hline DF1 & 3.4 & 250 & NF1 & 4.0 & 265 \\
\hline DF2 & 3.3 & 245 & NF2 & 3.7 & 260 \\
\hline DF3 & 3.5 & 255 & NF3 & 4.2 & 268 \\
\hline DF4 & 3.6 & 255 & NF4 & 3.8 & 270 \\
\hline DF5 & 3.5 & 250 & NF5 & 4.2 & 265 \\
\hline DF6 & 3.8 & 260 & NF6 & 3.8 & 260 \\
\hline DF7 & 3.3 & 245 & NF7 & 3.7 & 260 \\
\hline DF8 & 3.4 & 250 & NF8 & 3.7 & 260 \\
\hline DF9 & 3.5 & 256 & NF9 & 3.8 & 262 \\
\hline DF10 & 3.6 & 256 & NF10 & 3.8 & 262 \\
\hline DF11 & 3.7 & 256 & NF11 & 4.0 & 266 \\
\hline DF12 & 3.8 & 260 & NF12 & 3.6 & 258 \\
\hline DF13 & 3.5 & 255 & NF13 & 4.1 & 268 \\
\hline DF14 & 3.6 & 256 & NF14 & 3.6 & 258 \\
\hline DF15 & 3.7 & 256 & NF15 & 3.8 & 270 \\
\hline DF16 & 3.8 & 260 & NF16 & 4.1 & 268 \\
\hline DF17 & 3.3 & 245 & NF17 & 3.7 & 260 \\
\hline DF18 & 3.4 & 251 & NF18 & 4.0 & 265 \\
\hline DF19 & 3.3 & 245 & NF19 & 4.2 & 268 \\
\hline DF20 & 3.6 & 256 & NF20 & 3.6 & 258 \\
\hline DF21 & 4.4 & 279 & NF21 & 4.5 & 280 \\
\hline DF22 & 4.6 & 280 & NF22 & 4.5 & 280 \\
\hline DF23 & 4.5 & 280 & NF23 & 4.6 & 280 \\
\hline DF24 & 4.6 & 279 & NF24 & 4.5 & 280 \\
\hline DF25 & 4.6 & 279 & NF25 & 4.5 & 279 \\
\hline DF26 & 3.8 & 262 & NF26 & 4.2 & 268 \\
\hline DF27 & 3.7 & 257 & NF27 & 3.9 & 267 \\
\hline DF28 & 3.8 & 264 & NF28 & 4.1 & 268 \\
\hline DF29 & 3.9 & 266 & NF29 & 4.2 & 268 \\
\hline DF30 & 4.4 & 278 & NF30 & 3.9 & 268 \\
\hline DF31 & 3.8 & 265 & NF31 & 4.0 & 266 \\
\hline DF32 & 3.8 & 264 & NF32 & 4.1 & 266 \\
\hline DF33 & 3.8 & 264 & NF33 & 4.1 & 268 \\
\hline DF34 & 3.9 & 268 & NF34 & 4.1 & 267 \\
\hline DF35 & 3.8 & 265 & NF35 & 4.2 & 272 \\
\hline DF36 & 4.5 & 280 & NF36 & 4.1 & 268 \\
\hline DF37 & 4.0 & 270 & NF37 & 4.1 & 268 \\
\hline DF38 & 3.9 & 267 & NF38 & 4.0 & 267 \\
\hline DF39 & 3.8 & 265 & NF39 & 3.8 & 265 \\
\hline
\end{tabular}


Mycological analysis and potential health hazards of dextrose intravenous.....

\begin{tabular}{llllll}
\hline DF40 & & & & & \\
DF41 & 3.5 & 279 & NF40 & 41 & 270 \\
DF42 & 3.7 & 256 & NF42 & 4.9 & 268 \\
DF43 & 3.7 & 260 & NF43 & 3.8 & 270 \\
DF44 & 3.6 & 255 & NF44 & 4.0 & 268 \\
DF45 & 3.6 & 255 & NF45 & 4.1 & 270 \\
DF46 & 3.6 & 255 & NF46 & 3.9 & 270 \\
DF47 & 3.6 & 254 & NF47 & 4.0 & 268 \\
DF48 & 3.7 & 259 & NF48 & 3.8 & 266 \\
DF49 & 3.6 & 255 & NF49 & 4.5 & 280 \\
DF50 & 3.9 & 268 & NF50 & 4.6 & 281 \\
& 3.8 & 264 & & & \\
\hline
\end{tabular}

Detected Range (pH: 3.3 - 4.6; Osmolarity: 245 - 280 mosmol/l ) Normal Values ( pH:4.5 - 5; Osmolarity 280 mosmol/l)

Table 5: The pH and Osmolarity values of the $10 \%$ Dextrose intravenous fluids.

\begin{tabular}{|c|c|c|c|c|c|}
\hline $\begin{array}{l}\text { Visibly } \\
\text { defective }\end{array}$ & p H & $\begin{array}{c}\text { Osmolarity } \\
\left(\text { mosmol l-1 }^{-1}\right)\end{array}$ & $\begin{array}{l}\text { Visibly } \\
\text { Normal }\end{array}$ & p H & $\begin{array}{l}\begin{array}{l}\text { Osmolarity } \\
\left(\text { mosmol I I }^{-1}\right)\end{array} \\
\end{array}$ \\
\hline DT1 & 3.8 & 480 & NT1 & 3.9 & 525 \\
\hline DT2 & 3.8 & 482 & NT2 & 4.3 & 530 \\
\hline DT3 & 3.8 & 485 & NT3 & 4.2 & 535 \\
\hline DT4 & 4.2 & 534 & NT4 & 4.2 & 545 \\
\hline DT5 & 3.8 & 482 & NT5 & 4.2 & 545 \\
\hline DT6 & 4.0 & 525 & NT6 & 4.2 & 545 \\
\hline DT7 & 4.0 & 525 & NT7 & 3.9 & 525 \\
\hline DT8 & 3.8 & 480 & NT8 & 3.9 & 525 \\
\hline DT9 & 3.8 & 480 & NT9 & 4.3 & 530 \\
\hline DT10 & 4.2 & 535 & NT10 & 4.3 & 530 \\
\hline DT11 & 4.2 & 535 & NT11 & 4.1 & 528 \\
\hline DT12 & 4.0 & 525 & NT12 & 4.2 & 545 \\
\hline DT13 & 3.8 & 480 & NT13 & 4.1 & 528 \\
\hline DT14 & 3.9 & 520 & NT14 & 4.3 & 530 \\
\hline DT15 & 3.8 & 480 & NT15 & 4.1 & 528 \\
\hline DT16 & 3.8 & 480 & NT16 & 3.9 & 525 \\
\hline DT17 & 4.0 & 525 & NT17 & 4.2 & 535 \\
\hline DT18 & 3.8 & 482 & NT18 & 3.9 & 525 \\
\hline DT19 & 4.2 & 534 & NT19 & 4.1 & 528 \\
\hline DT20 & 3.9 & 520 & NT20 & 4.3 & 530 \\
\hline DT21 & 3.8 & 482 & NT21 & 4.2 & 545 \\
\hline
\end{tabular}

Detected range ( pH: 3.8 - 4.3; Osmolarity: 480- 545 mosmol /l) Normal values (pH: 6; Osmolarity: 555mosmol/ l)

Parenteral dosage form must be sterile right from the point of leaving the factory to the moment of administration to the patient. This inherent property of parenteral pharmaceuticals is imparted into the product during the manufacturing process by rigidly adhering to sound and validated procedures at every steps of the preparation ( Levchuk, 1991). However, this is not obtained in some cases thereby causing economic losses to manufacturers and health hazards to users. In this work, fourteen (14) fungal species belonging to Ten (10) genera were detected. Statutory pharmaceutical authorities stated that no living microorganism or their products must be present in sterile pharmaceutical products. However, ninety percent $(90 \%)$ of the total samples failed to comply with the test for sterility. While the highest fungal count of $1600 \mathrm{cfu} / \mathrm{ml}$ was obtained in the defective sample DT1, the least count was $10 \mathrm{cfu} / \mathrm{ml}$ in the visibly normal NF2 while NF17 and NF19 had 20cfu / ml; NF8 and NF9 had $30 \mathrm{cfu} / \mathrm{ml}, \mathrm{NF} 10$ and NF12 had $40 \mathrm{cfu} / \mathrm{ml}$ each (Table 1b). The total fungal counts and diversity in the visibly defective samples exceeded the visibly normal samples of all products tested and this may be conclusive of the visible defects in the defective samples (Table 1a,1b,1c,1d). 
In a previous study, Atata et al., 2007 reported Aspergillus spp and Penicillium spp in some Dextrose intravenous fluids in Nigeria. However, in this study, the predominant fungal contaminants were Aspergillus repens, Candida krusei, Geomyces cretacea, Aspergillus glaucus, Penicillium roqueforti, Chaetomium spp, Aspergillus japonicus, Exophiala dermantitidis, Fusarium oxysporium, Candida valida, Candida parapsilosis, Paecilomyces variotii and Geotrichum candidum. While spoilage organisms were recovered more from the defective samples, pathogenic strains were recovered from the seemingly normal samples of both the 5\% and $10 \%$ Dextrose intravenous fluids (Tables 2 and 3 ).

Considering the degree of proliferation and degradation that may occur in a product, the relationship between the fungal counts and the shelf life of such product remains an important factor. With respect to the period of stay on the shelf and the fungal population, samples DF41>DF27 > DF12>DF8 $>$ DF7 $>$ DF3 $>$ DF5 $>$ DF35> DF34 (Table 1a); NF31 >NF32>NF21 > NF14> NF7>NF36>NF34>NF49>NF48> NF47 (Table 1b); DT1>DT2>DT4> DT3>DT6>DT13>DT18>DT20 (Table 1c); NT3>NT2> NT18> NT17> NT14> and NT15> NT13 (Table 1d); these indicate that the longer the product was kept before use, the higher the fungal counts. Though none was expired prior to analysis, it is apparent that long storage periods allowed the initially undetected viable spores to multiply, proliferate and become detectable. Therefore the closer the time of use to the manufacturing date the better; but the closer the time of use to the expiry date the worse for the drug and the user.

The microbial contaminants in a pharmaceutical preparation may induce disease in the consumer without necessarily producing spoilage of the product. There are two possible mechanisms for this; either infection by the pathogenic organism or through toxins liberated into the product (Ringertz and Ringertz,1982).The risk of microorganism producing an infection is dependent on the species, dose administered, route of administration and susceptibility of the host. However, irrespective of the innoculum density, in as much as the products are administered directly into the blood circulation, the presence of any microorganism or their product is crucial. It should also be noted that an organism does not need to proliferate in the product in order to induce disease, it just needs to survive ( Hugo and Russell, 1992 ). Acute systemic infections and death have resulted from the administration of microbially contaminated intravenous infusions. The organisms most frequently causing septicemia are Candida, Aspergillus and Paecilomyces (Goldman et al., 1993; McQuillen et al., 1994 ). In this work, species of these genera, namely, Candida valida, Candida krusei, Candida parapsilosis, Paecilomyces variotii, and Aspergillus japonicus were recovered from the visibly normal samples. Therefore, the use of these infusions has the potential of being injurious to health.

In addition to fungemia and septicemia, these fungi may liberate some pyrogenic substances in the intravenous infusions. Absorbtion and dissemination by the blood of pyrogens can be accompanied by severe clinical symptoms such as low fibrinogen level, hypotension, acute shock and eventual death. Metabolites produced during fungal growth include mycotoxins which are associated with a number of diseases in man and animals. Mycotoxins are produced by a number of species such as Penicillium and Fusarium which were typically identified in this research. Several Fusarium species produce fumonisins and trichothecenes while several Aspergillus and Penicillium species produce ochratoxins. Aflatoxin by species of Aspergillus is known to be a potent hepatocarcinogen (Nigam et al., 1994 ). Candida krusei, Aspergillus and Paecilomyces constituted the highest proportion in both the defective or normal samples. Exophiala, Geotrichum and Chaetomium were only found in the defective 10\% Dextrose injections while Fusarium and Candida were recovered from the normal samples of both category of products. The growth of these organisms may have been favored by their capability to withstand high osmotic tension of the high sugar concentration of the fluids; and the high temperature $\left(25^{\circ} \mathrm{C}-40^{\circ} \mathrm{C}\right)$ of storage of the fluids.

The $\mathrm{pH}$ of blood is 7.4 and blood plasma osmolarity is $306 \mathrm{mosmol}^{-1}$. It is mandatory that parenteral intravenous injections must be in this range. Five percent ( $5 \%$ ) Dextrose intravenous injection has a pH range between 4.5 and 5 . As a result of growth and metabolic activities of the fungal contaminants, there were corresponding reductions in $\mathrm{pH}$ of the products emanating from production of acids by the fungi (Table 3 ).

The $\mathrm{pH}$ was reduced to 3.3 in samples DF2, DF7, DF17, and DF19; 3.4 in samples DF1, DF8, and DF18; 3.5 in samples DF3, DF5, DF9, and DF13; 3.6 in samples DF4, DF10, DF14, DF20, NF12, NF14, and NF20; 3.8 in samples NT1, NT2,NT3 and DF6 respectively. Any reduction below the critical safe level of pH 4 as in samples DF1, DF2, DT1, NF2, NF4, NF6, NF7, NF8, NF9, NF10, NF12, NF15, NF14, NF20, NF17, NT1, NT7, NT8, NT16 and NT18 (Tables 4 and 5) will potentially cause complications. The complications may include severe pain at the site of injection, tissue necrosis (De Lucia and Rapp, 1982 ), damage of venous endothelium and infusion phlebitis. Small changes in $\mathrm{pH}$ can signify large changes in $\mathrm{H}+$ ion concentration. Most importantly, the continuous infusion of hypotonic and acidic injections may overtax the buffering capacity of the blood. The incidence of phlebitis increases as infusate $\mathrm{pH}$ and osmolarity differs from that of blood (Marc , 2005).

In addition, as a result of microbial metabolism of the nutrients, the $5 \%$ dextrose injections were rendered hypotonic. There were corresponding reductions in osmolarity as the $\mathrm{pH}$ also reduced. Five percent 
$(5 \%)$ dextrose intravenous injections are isotonic solutions having osmolarity of $280 \mathrm{mosmol}^{-1}$, used for replacement of lost fluid and electrolyte imbalance in patients. Ten percent $(10 \%)$ dextrose injections are hypertonic solutions of osmolarity $555 \mathrm{mosmol}^{-1}$ for treatment of patients in hypoglycaemic coma, for energy supplementation and parenteral nutrition ( The Pharmaceutical Codex, 1994). Current USP recommendations for labeling of intravenous fluids require that osmolarity be stated on the package.

The result shows that the osmolarity of the $5 \%$ Dextrose injections had been reduced to 250 in sample DF1; 245 in samples DF2, DF7, DF17 andDF19; 265 in sample NF1, 260 in NF2 and 270 in NF4. In the 10\% Dextrose injection, the osmolarity had been reduced to 480 in defective samples DT1, DT8, DT9, DT13, DT15 and DT16; 482 in DT2, DT5, DT18, and DT21; 525 in Normal samples NT1, NT7, NT8, NT16, and NT18; 528 in NT11, NT13, NT15 and NT19; 530 in NT2,NT9, NT10, NT14 and NT20 (Tables 4 and 5 ). Therefore, the level of constituents and attributes as claimed by the product labels are often considerably lower than the normal range as at the time of presentation to users.

The intravenous administration of hypotonic solutions such as in the visibly normal samples NF1, NF2, NF4, NF6, NF7, NF8, NF9, NF10, NF12, NF14, NF17, NF18, NF19, and NF20, will result in fluid movement into the more concentrated venous endothelial cells and blood cells, elicit swelling of erythrocytes and haemolysis, thereby potentiating haemolytic anaemia.

Fungal infection are a particular concern in patients receiving a high concentration of Glucose in intravenous hyperalimentation (Schulhof, 2009) such as in the 10\% Dextrose injection in this study, which yielded Fusarium oxysporum, Candida parapsilosis and Candida krusei. Furthermore, the findings of this present study corroborates the earlier findings of Gupta et al., 2000 who detected Aspergillus fumigatus, Aspergillus niger and Candida albicans from dextrose intravenous infusions and concluded that the administered contaminated fluids in a rural setting was found to be a risk factor for development of endogenous fungal endophthalmitis.

Intraluminal spread of infection may result from intrinsic contamination of the infusion fluids. The apparent occurrence of the pathogenic fungi such as Aspergillus, Candida, Fusarium and Paecilomyces especially in the visibly normal samples coupled with significant reductions in $\mathrm{pH}$ and osmolarity values, suggest that these products are of potential health hazards. The manifestation of such hazards is highly probable especially in developing countries where "commercially sterile "products are often assumed to be actually sterile and are therefore consumed or administered without adequate precautions in respect of debilitated patients whose immunity are already compromised by illness. The patients may experience severe lifethreatening complications.

These factory produced pharmaceuticals may constitute reservoirs for disseminating potentially dangerous infections to the community. Consequently, morbimortality and cost of healthcare will increase.

This research is a contribution to post- marketing surveillance, which Drug Regulatory Authorities have recently expressed frustration at not being able to dedicate more resources (WHO, 2006). The results are indices of substandard medicines which represent a far larger risk to public health than counterfeit medicines.

Most importantly, mortality in patients receiving intravenous infusion / therapy are not usually investigated in developing countries but commonly attributed to the underlying ailment or circulatory overload. For example, the fever often observed after post - surgery administration of intravenous fluids is usually interpreted to reflect a general depreciation of the patient's condition rather than possible presence of pyrogens or endogenously contaminated fluids.

The use of contaminated intravenous fluid predisposes patients to increased risk of Hospital acquired infections and extended hospitalization. In Africa, research on intravenous therapy cannot be over-emphasized, considering the developing nature of our economies, scientific technologies, and the enormity of the potential hazards associated with contaminated intravenous fluids. Currently, there are no technologies yet to detect endogenous contamination of fluids either post marketing or at the point of administration, apart from the visual detection of defects in the aesthetic qualities of these products. Therefore, the presence of any form of microorganism in intravenous fluid is clearly a threat to life. This investigation, in addition to other reports, suggest that the current design and / or protocol for sterilization of these products may not be safe or adequate for patients' use. The recommended use of in - line filters will neither filter out the pyrogens, nor be selective for the reduced osmolarity and $\mathrm{pH}$. Consequently, existing standards and procedures should be reviewed and amended accordingly in order to produce a perfectly safe, wholesome and improved products in developing countries. 

manufactured in Kenya. East African Medical Journal 72 (12): 800-803.

[2]. Atata, R. F., Ibrahim, Y. K., Akanbi, A. A., Abdul, I. F., Sani, A., and Ahmed, R. N. (2007) Microbiological quality of some brands of intravenous fluids produced in Nigeria. African J. of Biotech. 6 (19) : 2197 - 2200.

[3]. British Pharmacopoeia (1988)Test for sterility and microbial contamination.Appendix XVIA, HMSO, London.pp-A191A198.

[4]. Caudron, J.-M., Ford, N., Henkens, N., Mace, C., Kiddle- Monroe, R. and Pinel, J. (2008) Substandard medicines in resource - poor settings : a problem that can no longer be ignored . Tropical Medicines and Intern. Health. 13 (8) : $1062-1072$.

[5]. Cornelius, J., Yue, Yu, Alfred, L. and Hong, M. (1998) Inhibition of RNA synthesis as a therapeutic strategy against Aspergillus and Fusarium : Demonstration of in vitro synergy between Rifabutin and Amphotericin - B. Antimicrobial Agents and Chemotherapy $42(93): 509-513$

[6]. Coulombe, R. A. (1993) Biological actions of mycotoxins. Journal of Dairy Science 76 : 880 - 891

[7]. Curry, C. R. and Quie, P.G. (1971) Fungal septicemia in patients receiving parenteral hyperalimentation. New England Journal of Medicine $\mathbf{8 5}: 1221$.

[8]. De Lucia, P.P., Rapp, R. P. (1982) Parenteral drug delivery system. In Pharmaceutical and Pharmacy Practice. Banker G.S. and Chalmers, R.K. (eds.) Lappinscott, Philadephia. Pp238-278.

[9]. Elamin, S., Al-Amin, M., Hashim, A., Amin, A., Nasereldeen, S. , et al.,( 2010) Outbreak of peritonitis in a continuous ambulatory Peritoneal Dialysis population following the use of contaminated Peritoneal Dialysis Fluids. Arab J. of Nephrology and Transplantation 3(2): $47-52$

[10]. Goldman, M., Ottage, J.C. and Weaver, D.C. (1993) Candida krusei fungemia. Report of four cases and Review of literature. Medicine $72: 143-150$

[11]. Gupta, A., Gupta, V., Dogra, M., Chakrabarti, A., Ray, P., Ram, J., and Patnaik, B.(2000) Fungal endophthalmitis after a single intravenous administration of presumably contaminated dextrose infusion fluids. Retina 20 (30) : 262- 268

[12]. Harrigan ,W.F. and McCance, M.F. (1976) Laboratory methods in food and Dairy Microbiology. $7^{\text {th }}$ edn. Churchill Livingstone, New York. pp $250-262$

[13]. Hugo, W.B. and Russell, A.D. (1992) Microbial contamination and spoilage of Pharmaceutical products. In Pharmceutical Microbiology. Hugo and Russell (eds.) $5^{\text {th }}$ edn. Blackwell Scientific Publications London.pp286 - 350.

[14]. Kumar, S., Baradkar, V., De, A. and Mathur, M. (2011) Outbreak of systemic candidosis associated with contaminated parenteral fluids. Bombay Hospital Journal 53 (1)

[15]. Kuwahara, T., Kaneda, S., Shimono, K., and Inoue, Y. (2010) Growth of microorganisms in total parenteral nutritions without lipids. Intern. J. of Medical Sciences 7 (1): 43

[16]. Levchuk, J.W. (1991) Good Manufacturing Practices and Clinical supplies. Journal Parenteral Science Tech. 45: 152

[17]. Lodder, J. (1970) The Yeasts. A taxonomic study. $1^{\text {st }}$ edn. Amsterdam, Holland

[18]. Macias, A. E., Martha, H., Samuel Ponce de L., Munoz, J. M., Chavez, R. A., et al. (2010) Contamination of intravenous fluids : A continuing cause of hospital bacteremia. American Journal of Infect. Control. 38 (3): 217 - 221

[19]. Marc Stranz (2005) The implications of Osmolarity, Osmolality and p H in infusion Therapy. INS Proceedings (May) 1 - 5

[20]. Marzec, A.L. and Heron, L. G. (1993) Paecilomyces variotii in peritoneal dialysate. Journal of Clinical Microbiology 31: $2392-2395$

[21]. Mcquillen, P., Zingman, S., Meunier, F. and Levitz, L. (1994) Invasive infection due to Candida krusei. Clinical Infec. Diseases $14: 472-478$.

[22]. Nigam, S.K., Ghosh, S.K. and Malaviya, R. (1994) Aflatoxin, its metabolism and carcinogenesis : a historical review. Journal of Toxicology-Toxin Reviews 13: 179 - 203

[23]. Peggy Gargis (2011) Nine hospital deaths related to contaminated IV fluids. Dangerous drug recalls. Reuters News

[24]. Public Health Laboratory Services Working Party (1971) Microbial contamination of medicinal preparations administered to Hospital patients. Pharm. Journal $207: 96-99$

[25]. Polin, R.A. and Siman, S. (2003) Nosocomial infections in the Neonatal Intensive Care Unit. NeoReviews 4 (3) 81 - 89

[26]. Rajalakshmi, T.K.(2011) A dying system Frontline 28(7) 1 -3

[27]. Rao, S. and Ali, U. (2005) Systemic fungal infections in Neonates. J. Postgrad Med. 51 (5) 27 -29

[28]. Ringertz, O. and Ringertz, S. (1982) Effects of microbial contaminants in Pharmaceuticals. Advances in Pharmaceutical Science 1 : $201-225$.

[29]. Schulhof, Steven (2009) Intravascular catheter associated infections. In Total Parenteral Nutrition. TPN Pdf pp 3-7.

[30]. Scott, E. M., Gorman, S.P., Wyatt, T.D. and Magill, E.A. (1985) Growth of microorganisms in Total Parenteral Nutrition mixtures and related clinical observations. Journal of clinical and hospital Pharm. $10: 79-88$.

[31]. The Pharmaceutical Codex (1994) Principle and Practice of Pharmaceutics. Walter, L. (ed.) The Pharmaceutical Press. London. Pp $45-70$

[32]. The U.S. Pharmacopoeia (2008) Available at http://www.uspdqi.org/pubs/other/GHC- Drug Quality Matrix.pdf

[33]. Vagnar, B. and Henao, N.A. (2011) Infection of the CNS by Candida. J. Infectious Diseases and Immun. 3 (5): $79-84$

[34]. W.H.O (2006) WHO Consultation on Regulatory Technical Package and Model for Regulatory Decision Making. WHO, Geneva.

[35]. Zarrin, M., and Zarei, M. A. (2010) Central Nervous System Fungal infections: a review article. Judishapur J. Microbiol. 3 (2): $41-47$ 